Note: This is an accepted manuscript version of an article published by Taylor \& Francis Group in the Journal of Aggression, Maltreatment and Trauma on 25/09/2014, available online:

http://www.tandfonline.com/10.1080/13669877.2013.815649

The final publication is available at Taylor \& Francis via http://dx.doi.org/10.1080/10.1080/13669877.2013.815649

Citation: Knuth, Daniela, Kehl, Doris, Galea, Ed, Hulse, Lynn, Sans, Jordi, Vallès, Lola, Roiha, Malin, Seidler, Frank, Diebe, Eberhard, Kecklund, Lena, Petterson, Sara, Wolanin, Jerzy, Beltowski, Grzegorz, Preiss, Marek, Sotolárová, Marie, Holubová, Marketa, Sofuoglu, Turhan, Sofuoglu, Zeynep Baskaya, Pietrantoni, Luca, Saccinto, Elisa and Schmidt, Silke (2014) BeSeCu-S - a self-report instrument for emergency survivors. Journal of Risk Research, 17 (5). pp. 601-620. ISSN 1366-9877 (Print), 1466-4461 (Online) (doi: $\underline{10.1080 / 13669877.2013 .815649 \text { ) }}$ 


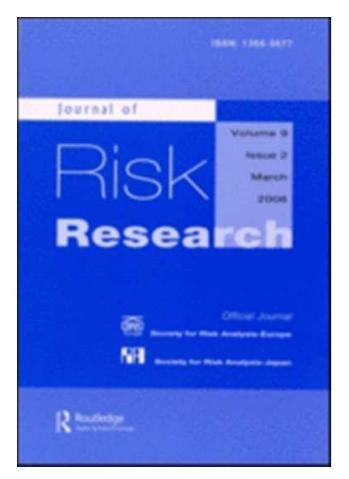

\section{BeSeCu-S - A self-report instrument for emergency survivors}

\begin{tabular}{|r|l|}
\hline Journal: & Journal of Risk Research \\
\hline Manuscript ID: & RJRR-2012-0076.R2 \\
\hline Manuscript Type: & Original Article \\
\hline Keywords: & emergency, behaviour, survivors \\
\hline & \\
\hline
\end{tabular}

SCHOLARONE ${ }^{\mathrm{M}}$

Manuscripts 


\section{Background}

In recent years the field of disaster research has received more attention. As with man-made incidents like the bombings in Madrid 2004 and London 2005, the impact of natural disasters has been very severe. Europe has faced incidents like the floods of 2002 and 2010 in the Czech Republic, Germany and Poland, as well as the earthquakes in Italy in 2009 and Turkey in 1999 and 2010. In 2010, natural disasters caused more than 297,000 deaths worldwide and another 217,000,000 people were affected (Guha-sapir, 2010). However, other, smaller-scale emergency events such as building fires, particularly fires in domestic buildings, have also posed significant harm to people and property (Kobes \& Groenewegen, 2009).

Different instruments have been developed over the years in order to investigate responses to a traumatic, fearsome event that threatens lives. The Peritraumatic Distress Inventory (PDI; Brunet et al., 2001) has 13 items and focuses on emotional reactions as well as physiological stress reactions during the event. Dissociative reactions during an event like feelings of unreality, being detached from oneself, being confused or a change in sense of time can be assessed with the 10 items of the Peritraumatic Dissociative Experiences Questionnaire (PDEQ; Marmar, Weiss, \& Metzler, 1996). A meta-analysis (Ozer, Best, Lipsey, \& Weiss, 2003) revealed subjective psychological responses to an event like perceived life threat and peritraumatic emotions as being important predictors of the later occurrence of Posttraumatic Stress Disorder (PTSD). In order to investigate posttraumatic stress following traumatic events, the Impact of Event Scale (IES) was developed (Horowitz, Wilner, \& Alvarez, 1979). The self-report instrument was revised by Weiss and Marmar (1997; the IES-R) and comprises the three subscales intrusion, avoidance and hyperarousal. Like the aforementioned peritraumatic measures, the IES-R assesses responses to the event in general, rather than responses to any specific aspects or moments relating to the event. 
Another similarity is that its items focus mainly on emotional, physiological, and cognitive responses to the event, albeit ones occurring at a later point in time.

In events like terrorist attacks, floods, earthquakes and building fires there is another type of peri-event response that is important: victims' activities. What individuals do when faced with a threat and in the moments afterwards can directly influence their chances of escaping danger and reaching a place of safety. Studies of behavioural responses during a disaster or smaller-scale emergency that threatens lives and property, and especially during a building evacuation, have usually been investigated in a single type of event (Kasapoglu \& Mehmet, 2004; McConnell, Boyce, Shields, Galea, Day, \& Hulse, 2010; Proulx \& Reid, 2006; Zhao, Lo, Zhang, \& Liu, 2008). Previous investigations have often focused on initial activities like searching for more information or ignoring threat cues and continuing with one's current task (e.g. Samochine, Boyce, \& Shields, 2005) as well as evacuation preparations such as collecting belongings (e.g. McConnell et al., 2010). Activities undertaken during this "response phase" (Galea, 2009) can not only delay the start of an individual's evacuation from a structure (Zhao et al., 2009) but also affect their risk of incurring physical injury (Glenshaw, Vernick, Frattaroli, Brown, \& Mallonee, 2008) and have been found to be influenced by factors like emotional distress (Zhao et al., 2009), perceived cues to the threat (Zhao et al., 2009) and prior emergency knowledge (Glenshaw et al., 2008; Zhao et al., 2009). Perceived control and urgency may possibly even be involved (Glenshaw et al., 2008; Zhao et al., 2009). However, such studies have highlighted a temporal factor, with victims' responses changing at different moments during the event (Glenshaw et al., 2008; McConnell et al., 2010; Zhao et al., 2009).

It would seem then that in order to better understand the effects of peri-event responses on well-being following a disaster or similarly threatening emergency situation, victims' emotions (i.e. feelings and physiological states), cognitions plus their activities 
would need to be investigated. Responses would also need to be investigated with respect to the different stages of the event. Such knowledge could be of use to professionals in various health and safety fields and help develop preventive and protective interventions. However, investigations of relationships between a fuller range of peri-event responses and post-event outcomes are very rare, as are investigations of relationships between the range of peri-event responses, and existing measures like the PDI and PDEQ would not be adequate for this task. One study (Kaysen, Morris, Rizvi, \& Resick, 2005) that did look at peri-event emotions, cognitions and activities - albeit for emergencies involving only a single victim (i.e. females who had been robbed, sexually or physically assaulted) - not only found that certain activities and emotions were associated with perceived threat, but also that peri-event responses could differ according to the type of event experienced. Although Kaysen et al. (2005) did not examine responses at specific moments during the events, they nevertheless noted a temporal factor (event duration) as being important. Such findings support the notion that a more comprehensive examination of responses to threatening events is needed.

There is one further point to consider. The rise of globalisation and multicultural nations, and the fact that future large-scale threatening events are likely to be multinational events (Lahad \& Crimando, 2010), prompt the question of whether culture will impact psychological and behavioural responses during disasters and other emergency situations. Most studies addressing this issue have been carried out in the UK, the USA, Canada or Australia (Briere \& Elliott, 2000; Brown, 2003; Kobes, Helsloot, Vries, \& Post, 2010; Lindell \& Perry, 2011); cultures that might be very similar. However some study results indicate that culture might have a possible influence on the behavioural response to a threatening event (Fothergill, Maestas, \& Darlington, 1999; Rodríguez, Quarantelli, \& Dynes, 2006) and thus requires proper attention. Yet a comparison between cultures, different types of events, as well as relationships between peri-event responses, is not possible since no standardised 
instruments have been used and research usually focuses on one particular event, therefore one can only make assumptions regarding this specific incident (Briere \& Elliott, 2000).

In order to investigate relationships between different peri-event responses and postevent outcomes, across different stages of an event and different event types, and also between different nationalities, the BeSeCu-S (Behaviour, Security, and Culture - Survivor) questionnaire was developed. This was as part of the wider EU-funded BeSeCu Project, with research conducted by partners in eight different countries in Europe. The BeSeCu-S questionnaire was designed for survivors of any event that threatened lives and property and meant evacuation of a structure was a valid option. Topics and questions were presented chronologically (i.e. relevant to pre-event, peri-event, then post-event factors) and collect data on a variety of variables related to the individual, to the event in question and to threatening events in general. It allows not only investigations of acute trauma-related constructs like emotional distress, panic attack symptoms, perceived threat and posttraumatic stress but also enables an investigation between these constructs and activities undertaken by individuals during the event. $\mathrm{BeSeCu}-\mathrm{S}$ displays the result of cooperation between practitioners and researchers, as demanded by different disaster researchers, in order to compare experiences and include future needs (Fischer, 2008; Kasapoglu \& Mehmet, 2004).

The BeSeCu-S was tested with over a thousand survivors from Germany, United Kingdom, Spain, Italy, Sweden, Poland, Czech Republic and Turkey. It was hypothesised that analyses would reveal the following outcomes: (1) The BeSeCu-S would be suitable for international use; (2) the $\mathrm{BeSeCu}-\mathrm{S}$ would be suitable for capturing the experiences of survivors across different event types and across different stages of an event; (3) survivors' peri-event responses (at least some emotional and cognitive responses) would be significantly associated with post-event stress; (4) peri-event responses (at least some activities) would be significantly associated with time to begin evacuating and injuries incurred; and (5) prior 
emergency knowledge as well as emotional and cognitive peri-event responses would be significantly associated with at least some peri-event activities.

\section{Method}

\section{Questionnaire development}

The questionnaire was developed in consecutive steps including qualitative and quantitative data approaches. In addition to the interdisciplinary $\mathrm{BeSeCu}$ team of researchers and endusers, national and international experts from different fields of security research as well as emergency evacuation and national accident investigation boards supported the process of item generation. A literature review revealed the main topics and preliminary structure for the focus groups and interviews with survivors and first responders. These sessions were carried out with 132 participants in all project-partner countries (i.e. Germany, United Kingdom, Spain, Italy, Sweden, Poland, Czech Republic and Turkey) and the most frequently reported answers were used in order to formulate a first set of items in relation to the aforementioned and newly formulated categories (Freitag, Grimm, \& Schmidt, 2011; Grimm, Hulse, Preiss, \& Schmidt, n.d.). A pre-test with a convenience sample of 336 participants as well as 11 cognitive debriefing task participants (see Collins, 2003; Eremenco, Cella, \& Arnold, 2005) was drawn in order to test feasibility, practicability and difficulty of items (Grimm, Hulse, \& Schmidt, 2012). As a result of these steps, a final questionnaire draft was developed in English; therefore a translation into all corresponding languages of all partner countries (i.e. German, Spanish, Italian, Swedish, Polish, Czech, and Turkish) was necessary using a forward-backward-forward translation technique (Hilton \& Skrutkowski, 2002; Petersen, Schmidt, Power, \& Bullinger, 2005). The questionnaires were identical with respect to layout and design and available online and as a paper-pencil version. Regarding recruitment, each project partner was responsible for recruiting a nationwide sample in their own country and 
therefore applying the appropriate recruitment strategies (Knuth, Kehl, Stegemann, \& Schmidt, in press).

\section{Participants}

Participants were eligible for the study if they gave informed consent and met the following inclusion criteria: (1) experienced one of the following events: domestic fire, fire in a public building, flood, earthquake or terrorist attack; (2) at least 18 years of age; (3) knew that the emergency services attended the incident; and (4) the incident was no longer than 11 years ago (i.e. occurred not earlier than 1999). Criterion number three regarding the emergency services was not mandatory for earthquake, terrorist attack and flood survivors since it can be assumed that the emergency services are always involved in such events even though survivors might not have been in direct contact with them. Criterion four was chosen in order to include survivors of the Izmir earthquake of 1999. Furthermore, research regarding memory biases has found that memories are less biased in children at the age of seven and older (Bauer, Burch, Scholin, \& Güler, 2007; Cordón, Pipe, Sayfan, Melinder, \& Goodman, 2004; Pillemer, 1998; Rubin, 2000) and since participants had to be at least 18 years of age, this meant the sample only included participants who were at least seven years of age at the time of the incident. A sample of 1130 survivors met the inclusion criteria. Table 1 presents the socio-demographic information of the participants $(N=1112)$ that experienced the incident in one of the partner countries. Participants who experienced an event in other countries like the USA, Malaysia or Japan were excluded. The mean age of the sample was 40.40 years $(S D=15.27)$ with ages ranging from 18 years to 96 years. The mean time since the event was 3.21 years $(S D=3.39)$.

\section{Measurement}


The final BeSeCu-S questionnaire combines the findings from the cross-cultural focus groups and interviews, literature review, expert consultation and pretesting (Knuth, Kehl, \& Schmidt, in press). A 5-point Likert scale ranging from 1 to 5 ( $1=$ not at all, $2=$ a little bit, $3=$ moderately, 4=quite a bit, 5=extremely) was adopted from the IES-R (Weiss \& Marmar, 1997) and used for all items of the emergency knowledge, emotional distress and perceived threat scales mentioned below, since translated versions already existed in the languages of participating countries (Bergh Johannesson, Stefanini, Lundin, \& Anchisi, 2006; Corapcioglu, Yargıç, Geyran, \& Kocabaşoğlu, 2006; Gargurevich, Luyten, Fils, \& Corveleyn, 2009; Juczyński \& Ogińska-Bulik, 2009; Maercker \& Schützwohl, 1998; Pielmaier \& Maercker, 2011; Preiss et al., 2004). The mean scores of these scales were calculated and used in analyses if at least $75 \%$ of the scale items were answered.

Select parts of the final $\mathrm{BeSeCu}-\mathrm{S}$ are discussed here. The first part concerns preevent variables of interest to this paper and the second peri- and post-event variables.

\section{Part I - Background information}

This section consisted of socio-demographic items (see Table 1). Migrant background was assessed with questions about the participant's country of birth, the participant's parents' country of birth, as well as the participant's citizenship (Schenk et al., 2006).

Additionally, participants' emergency knowledge prior to the event in question was addressed here with the Emergency Knowledge Scale (EKS). Participants were asked the following question: Before the incident occurred, what knowledge did you have that would be of use in an emergency? Seven different statements were used to assess emergency knowledge: I had professional knowledge, gained from working for the emergency services; I had first aid knowledge, gained from a first aid course; I had fire safety knowledge, gained from being a warden/fire safety officer; I had taken part in fire drills at school; I had taken 
part in fire drills at work; I had read safety notices/evacuation plans in public places, such as in hotel rooms, train carriages, etc.; and I had thought about what would happen if an emergency occurred in such a location and had prepared my own evacuation plan.

\section{Part II - The specific incident}

Questions concerning the event were subdivided into four different stages: 1. Beginning, 2. Realisation, 3. Evacuation and 4. Aftermath.

1. Beginning. The first stage related to the moment just prior to participants perceiving cues to the threat. Emotional distress was assessed as a proposed baseline level with respect to this stage using the Emotional Distress Scale (EDS). Participants were asked: Before the incident occurred, what were your feelings? and rated their emotional distress with respect to the following items: I was nervous, I was scared, I was upset, I felt stress and I was calm (reverse coded).

2. Realisation. The second stage related to the time from when participants perceived cues to the threat and realised something was happening. Emotional distress was evaluated again using the EDS with respect to this stage now. It was also of interest to look at participants' responses with more emphasis on their physiological state. Thus participants were asked whether they experienced the 13 symptoms (i.e. fast heartbeat, sweating, trembling/shaking, shortness of breath, feeling of choking, chest pain or discomfort, nausea or abdominal distress, feeling dizzy, feelings of unreality or being detached from oneself, fear of losing control or going crazy, fear of dying, numbness or tingling sensations, chills or hot flushes) from the DSM IV (American Psychiatric Association, 2000) criteria for a panic attack. It was thought that such symptoms would be most evident at this stage of the event and so participants were only asked about this here. The sum of experienced symptoms was used as a score. 
The subjective evaluation of threat was assessed with the Perceived Threat Scale (PTS). Participants were asked the following two questions which were combined for the PTS: When you realised you were in an emergency situation, did you think your own life was in danger? and When you realised you were in an emergency situation, did you think the lives of your family/friends were in danger?

Perceived control was assessed with the following question: When you realised you were in an emergency situation, did you think you were able to deal with the situation?, which was rated on the aforementioned 5-point Likert scale.

Sense of urgency was primarily measured with a question about perceived time pressure (Did you feel pressure to act fast (e.g. felt the situation could worsen at any moment)?,yes/no). A second question (How would you describe your behaviour when you understood something was happening?) examined the sense of urgency further by exploring whether participants initially reacted in a more automatic manner, e.g. reverting to habitual behaviour, or whether they reacted in a more conscious manner, acting after reasoned deliberation. The answer options were automatic/instinctive (reacted without thinking) or conscious/rational (thought first then reacted).

Initial activities were assessed as follows: What was the first thing you did when you understood something was happening? with the answer options: I did nothing for a while; I tried to alert, comfort or save others who might be threatened by the situation; I tried to inform others about my situation, to reassure or update them; I sought help from the emergency services; I sought shelter inside the location; I tried to protect my property; I gave up and let happen whatever was about to happen; I gathered items in preparation for evacuation; I actively sought further information; Other. Participants were asked to only choose one of these answers. 
3. Evacuation. The third stage concerned the individual's evacuation from the structure and was therefore only mandatory for participants that either self-evacuated or had been rescued during the event. These participants were asked the following question: Approximately how long did it take you to start evacuating/be rescued? The answer options were: Within 30 seconds; Within 2 minutes; Within 5 minutes; Within 10 minutes; Within 30 minutes; Within 1 hour; Within 5 hours; Within 12 hours; Within 24 hours; More than 24 hours. Additionally, emotional distress, perceived threat and perceived control were assessed twice: once with reference to the period as they were making their way out of the structure and again at the end of this process (i.e. upon exiting the structure).

4. Aftermath. The last stage contained questions regarding consequences of the event. Participants were asked: Did you incur any physical injuries during the incident? (yes/no).

In order to assess current posttraumatic stress symptoms resulting from the event, the IES-R (Weiss \& Marmar, 1997) was included. The 22-item self-report instrument asked participants to rate their distress level during the past seven days with respect to the specific incident they described in the questionnaire. A total IES-R score was derived by calculating the mean score on the 22 items.

\section{Data analysis}

Descriptive statistics were calculated. Scales were investigated using factor analyses (principal component analysis) with varimax rotation and Kaiser normalisation. The determinant of the $R$-matrix had to be greater than .00001 , while all Corrected Item-Total Correlation $\geq .3$ and reliability of each scale (Cronbach's $\alpha$ ) had to be at least .7 (Field, 2009). Confirmatory factor analysis was performed for each scale introducing the different nationalities as groups and using maximum likelihood estimation with AMOS. Goodness of fit for each scale can be investigated by a number of different parameters: the comparative fit 
index (CFI) with proposed cut off value of $\geq .95$ for good fit (Hu \& Bentler, 1999); the incremental fit index (IFI) and the goodness of fit index (GFI) with values $\geq .90$ indicating good fit (Meyers, Gamst, \& Guarino, 2005); the root mean square error of approximation (RMSEA) with values $\leq .06$ (Hu \& Bentler, 1999) or at least $\leq .10$ (Meyers et al., 2005) indicating good fit. After scale and construct validity analyses, Friedman tests, followed up with post hoc analyses using Wilcoxon signed-rank tests (with Bonferroni corrections applied), were conducted to assess and locate any significant variations for variables that were measured across three or more event stages. Finally, Spearman's rho, Mann-Whitney $U$, Kruskal-Wallis and Chi-Squared tests were conducted to assess relationships between pre-, peri- and post-event variables (note, the last five categories for the time to begin evacuating variable were merged as few people reported starting to leave after 30 minutes). Data analyses were carried out using SPSS 19.0 and AMOS 19 computer software.

\section{Results}

The mean number of missing percentages varied across the questionnaire with $3.79 \%$ for the stage Beginning, 2.69\% for the stage Realisation, $4.48 \%$ for the Evacuation stage and $5.63 \%$ for the last stage concerning the Aftermath.

\section{Scale analysis}

The investigation of scales with the factor analyses (principal component analysis) revealed a one-factor solution for all scales. With respect to these one-factor solutions, $46.55 \%$ of the total variance was explained in the EKS, $64.69 \%$ in the EDS and $80.55 \%$ in the PTS. Scales were also tested in a confirmatory factor analysis. Confirmatory factor analysis revealed very good values for the EDS $[\mathrm{RMSEA}=0.044$ (0.035-0.052), GFI $=0.948, \mathrm{CFI}=0.961, \mathrm{IFI}=0.961$, $\left.\chi^{2}=136.64, \mathrm{df}=48 ; p<.001\right]$. For the EKS the values were as follows: RMSEA $=0.050(0.040$ - 
0.055), $\mathrm{GFI}=0.900, \mathrm{CFI}=0.873, \mathrm{IFI}=0.876, \chi^{2}=390.39, \mathrm{df}=112 ; \mathrm{p}<.001$. Table 2 shows descriptive scale characteristics and internal consistency values for all scales including their re-assessment through different stages. Internal consistency values (Cronbach's $\alpha$ ) for the scales in the total sample were at least .7. These values were confirmed in the national subsamples for the EKS and EDS. The values of the PTS in the national samples of Spain and the UK were below the value of .7.

\section{Discriminant and convergent validity}

Discriminant validity was tested in all scales with respect to participants' current general health status and weeks since the incident given that these measures were conceptually different from the described scales (Brunet et al., 2001). Discriminant validity was considered verified if the correlation coefficient was smaller than .3. As can be seen in Table 3, the EKS, EDS as well as PTS at any time of the event showed no or only small correlations with weeks since the event and health status. Exceptions in the national subsamples were as follows: EKS with health status in the Spanish sample $\left(r_{r h o}=-.37, p<.01\right)$; EDS with health status in the Swedish (Beginning: $r_{r h o}=.31, \mathrm{p}<.01$ ) and Spanish (Realisation: $r_{r h o}=.36, \mathrm{p}<.01$; During evacuation: $\left.r_{r h o}=.34, \mathrm{p}<.01\right)$ samples, and with weeks since the event in the Czech (Beginning: $r_{r h o}=.39, \mathrm{p}<.01$ ), Italian (Beginning: $r_{r h o}=-.30, \mathrm{p}<.01$ ) and Turkish (During evacuation: $r_{r h o}=.45, \mathrm{p}<.01$ ) samples; PTS with health status in the UK (Realisation: $r_{r h o}=.39$, $p<.05$; During evacuation: $r_{r h o}=.46, \mathrm{p}<.01$ ), Spanish (During evacuation: $r_{r h o}=.30, \mathrm{p}<.01$ ) and Swedish (During evacuation: $r_{r h o}=.33, \mathrm{p}>.05$ ) samples, and with weeks since the event in the Turkish (Realisation: $r_{r h o}=.45, p<.01$; During evacuation: $r_{r h o}=.45, \mathrm{p}<.01$; Upon exiting: $r h o=.59, \mathrm{p}<.01)$ sample.

For EDS and PTS, convergent validity was investigated with respect to the IES-R since the latter measure also assesses emotional and cognitive responses to a threatening 
event. The overall correlation between EDS during the incident and the IES-R was significant and ranged from $r_{r h o}=.57$ at realisation to $r_{r h o}=.64$ during the evacuation. For the PTS the correlation with the IES-R was significant and ranged from $r_{r h o}=.43$ upon exiting to $r_{r h o}=.52$ during the evacuation. In the national samples there were only a few exceptions for the PTS: in the Turkish (During evacuation: $r_{r h o}=.23, \mathrm{p}>.05$; Upon exiting: $r_{r h o}=.23, \mathrm{p}>.05$ ), UK (During evacuation: $r_{r h o}=.29, \mathrm{p}>.05$ ) and Swedish (Upon exiting: $r_{r h o}=.26, \mathrm{p}>.05$ ) samples the correlations were smaller and not significant. Furthermore, it was expected that the measure of panic-like symptoms and EDS at realisation would be tapping into related constructs; the correlations, in the total sample $\left(r_{r h o}=.60, p<.01\right)$ as well as in all subsamples $\left(r_{r h o}=.51-.74\right)$, supported this.

\section{Stage variations in emotion and cognition}

The EDS was administered across four event stages and a significant main effect was found, $\chi^{2}(3)=636.52, p<.001$. Specifically, emotional distress significantly differed from one stage to the next (Beginning vs. Realisation: $Z=-23.51, p<.001$; Realisation vs. During evacuation: $Z=-5.42, p<.001$; During evacuation vs. Upon exiting: $Z=-5.07, p<.001$ ), peaking at Realisation, then decreasing thereafter but always remaining above the level at the Beginning (see Figure 1). Perceived threat was measured at the latter three event stages and a main effect was revealed, $\chi^{2}(2)=482.74, p<.001$, with significant differences located between Realisation and During evacuation $(Z=-9.85, p<.001)$ as well as between During evacuation and Upon exiting $(Z=-14.95, p<.001)$. Like emotional distress, perceived threat peaked at Realisation and decreased thereafter. Perceived control, on the other hand, rose throughout the latter three event stages. A main effect was revealed, $\chi^{2}(2)=60.12, p<.001$, with the significant difference located between Realisation and During evacuation $(Z=-5.64, p<.001)$. 
The apparent increase in perceived control between During evacuation and Upon exiting was not significant $(Z=-1.73, p=.083)$.

\section{Relationships between pre-, peri- and post-event variables}

As mentioned earlier, some peri-event responses (emotional distress, perceived threat) were significantly associated with the measure of post-event stress, the IES-R. However, as Table 3 and further tests show, other peri-event variables were also significantly associated with the IES-R, namely panic-like symptoms, perceived control, time pressure $(Z=-8.53, p<.001)$ and the type of initial reaction $(Z=-9.10, p<.001)$. In other words, participants who, during the event, perceived themselves to experience more panic-like symptoms, be less in control, felt time-pressured and initially reacted in an automatic manner reported greater posttraumatic stress symptoms.

Tests revealed support for the other hypotheses too. Time to begin evacuating $\left(\chi^{2}[9]=45.90, p<.001\right)$ and Injuries incurred $\left(\chi^{2}[9]=38.22, p<.001\right)$ were significantly related to participants' initial activities (see Table 4). However, time to begin evacuating was also significantly related to another peri-event variable, PTS at Realisation (i.e. the greater the threat perceived, the quicker participants were in starting their evacuation; see Table 3). Moreover, being injured was also significantly related to participants perceiving themselves to have experienced greater distress $(Z=-2.76, p<.01)$ and threat $(Z=-4.18, p<.001)$, more panic-like symptoms $(Z=-5.51, p<.001)$ and less control $(Z=-3.45, p<.001)$ at Realisation, plus if they felt time-pressured $\left(\chi^{2}[1]=4.63, p<.05\right)$ and reacted in an automatic way initially $\left(\chi^{2}[1]=8.96, p<.01\right)$. Finally, initial activities were revealed to be significantly related to the EKS $\left(\chi^{2}[9]=43.95, p<.001\right)$, EDS (Realisation: $\left.\chi^{2}[9]=42.43, p<.001\right)$ and PTS (Realisation: $\left.\chi^{2}[9]=63.86, \quad p<.001\right)$. Panic-like symptoms $\left(\chi^{2}[9]=57.37, p<.001\right)$, perceived control $\left(\chi^{2}[9]=78.89, p<.001\right)$, time pressure $\left(\chi^{2}[9]=68.89, p<.001\right)$, as well as reacting either 
automatically or consciously $\left(\chi^{2}[9]=40.07, p<.001\right)$, were also significantly related to initial activities. Table 5 displays descriptive statistics relating to these variables and the initial activity undertaken by participants and shows that there was no one type of initial activity that was most related to all the different emotions and cognitions.

\section{Discussion}

This paper illustrates the content and psychometric properties of the $\mathrm{BeSeCu}-\mathrm{S}$, a comprehensive questionnaire for survivors. Project partners from eight different European countries elaborated the questionnaire specifically to learn more about human activities, emotions and cognition across cultures in relation to emergency situations. This process represents the first attempt to develop a standardised cross-cultural instrument for such interests. Survivors were not restricted to a specific incident (e.g. one particular fire or one particular flood), therefore this approach results in a more heterogeneous sample which increases the possibility of generalising findings with respect to different types of events (Briere \& Elliott, 2000).

Analyses confirmed that the questionnaire, on the whole, seemed to work as anticipated and captured what it was set out to capture. Scale analysis revealed one-factor solutions for the emergency knowledge, emotional distress and perceived threat scales, suggesting a unidimensionality of these scales. Internal consistency values (Cronbach's $\alpha$ ) for the scales were satisfactory for the total sample since they were at least .7, which is considered the limit for acceptable scales (Gliem \& Gliem, 2003). These values were also confirmed in each national and incident subsample except for the two-item PTS. The PTS had poor internal consistency values in the UK and Spanish subsamples and therefore this scale cannot be considered reliable for these subsamples. Since these items refer to the perceived threat to oneself and one's family, the results might be due to specific terrorist 
incidents in the UK and Spain. These events happened in the morning and mainly affected commuters. People commuting to and from work usually do not have friends or family around them and therefore should perceive no threat to them at the time of event. As a consequence, this scale might only be appropriate in other settings. Looking at the internal consistency values of the incident subsamples, this hypothesis is supported since these values are acceptable and good for domestic fires, floods and earthquakes but not for terrorist attacks and public building fires. Results of the confirmatory factor analysis suggest a very good fit in all indices for the EDS model. With respect to the other scales, CFI and IFI values were not satisfactory, whereas GFI and RMSEA values indicate at least an acceptable fit. Convergent and discriminant validity investigations suggest that the EDS especially can be considered valid in all national subsamples. The testing across different countries as well as different incidents largely supports our hypotheses (1) and (2) which stated that the BeSeCu$\mathrm{S}$ would be suitable for international use as well as for capturing the experiences in different event types. Furthermore, emotional distress, perceived threat and perceived control significantly differed between the stages investigated, highlighting the dynamic, temporal aspects of survivor responses and supporting the additional suggestion that the $\mathrm{BeSeCu}-\mathrm{S}$ would be suitable for capturing experiences across different stages of an event.

Hypothesis (3) was supported by significant relationships between the peri-event responses emotional distress and perceived threat with posttraumatic stress symptoms as measured by the IES-R. These relationships are in line with results from a meta-analysis concerning PTSD (Ozer et al., 2003). However, measures of panic-like symptoms, perceived control and sense of urgency were also significantly related to the IES-R suggesting researchers and clinicians should continue to consider a wide range of peri-event responses as being involved in the development of posttraumatic stress symptoms. 
Significant relationships between initial activities and the time to begin evacuating, the experience of being injured, prior emergency knowledge plus emotional and cognitive peri-event responses were not only in line with hypotheses (4) and (5) but also in line with previously found interactions (Glenshaw et al., 2008; Zhao et al., 2009). However, analyses revealed additional relationships between these variables, e.g. peri-event emotional or cognitive responses also being significantly related to how quickly survivors began moving away from the scene of danger and their success in avoiding physical harm. Any attempt to model causal relationships between pre-, peri- and post-event variables and to establish the relative size of their effects on one another is beyond the scope of this paper. Nevertheless, the data collected by the BeSeCu-S would allow for such attempts and more detailed analyses incorporating also possible effects of nationality, the type of incident and demographic factors will follow in subsequent papers.

The BeSeCu-S employs a retrospective self-reporting approach. Despite employing survey and interview techniques during the development of the instrument, one could nevertheless question if it is possible to obtain the desired information by self-report. Previous research regarding the relationship between emotional distress and memory differs greatly with respect to the type of event (emotional vs. neutral), type of information required (central vs. peripheral), time since the event (immediate vs. delayed), role in the event (witness vs. victim), way in which emotion was induced (through sudden shocking sights vs. thematically), and the type of recall (cued vs. free recall) (Bornstein, Liebel, \& Scarberry, 1998; Christianson \& Loftus, 1991; Christianson, 1992; Hulse, Allan, Memon, \& Read, 2007). Memories of disasters have been found to be very durable, even after two years (Stallings, 2007, p. 66). Although there was great variation in the time elapsed since the event in the current study (up to 11 years ago) the mean number of years elapsed since the event was just over three years and should be reasonable for assessing peri-event psychological 
responses at least (Brunet et al., 2001). Furthermore, the non-significant or weak correlations between the number of weeks since the event and other variables in the current study would indicate that participants' memories of their responses were not distorted by the passage of time. Experimental studies have concluded that for emotionally arousing events involving shocking sights such as horrific injuries, central details seem to be remembered better while peripheral information is remembered less well than in emotionally neutral events (Brown, 2003; Christianson \& Loftus, 1991). A further study, which investigated the reliability of delayed self-reports regarding experiences in a hurricane (Norris \& Kaniasty, 1992), found that, in particular, reports of losses and preparedness remained stable when compared to the first reports made ten months earlier. A small tendency toward recalling more information was only found with respect to social support (Norris \& Kaniasty, 1992). To conclude then, it has been shown that survivors are quite able to remember aspects of a distressing event, even after a long period of time, although longitudinal studies covering longer periods of time are very rare. Nevertheless it is important to note that some outcomes of an event (e.g. impacted life domains, support received or damage incurred) cannot be investigated immediately after the event.

The use of various sources for item generation is one of the strengths of the $\mathrm{BeSeCu}$ S. Not only were actual survivors of different threatening events included, but also experts from the field of disaster and security research and, just as importantly, practitioners, who could include their needs for specific information. Practitioners were included at all stages of the development process to achieve an increased interaction with researchers as demanded by scholars (Fischer, 2008). The different parts of the questionnaire (i.e. pre-, peri- and postevent) are a very distinctive and a unique attribute of the BeSeCu-S which allows the researcher to get information in relation to different phases. 


\section{Limitations of the study}

One limitation of the questionnaire might be the aim of its global usage. Although the questionnaire was designed with respect to five different event types, it might not be as applicable for one as for another. For flood incidents, the stages approach might be challenging. Since flooding is frequently caused and accompanied by heavy rain or bad weather conditions, it can be argued that the onset is therefore rather slow giving people more time to prepare and react. In addition, the duration of the incident itself can be much longer (days or weeks even) than for the other events. Questions regarding the exact date, time, start of evacuation and even emotional distress at the different event stages are more difficult to answer since each stage might take several days in itself. Additionally, the focus of this questionnaire concerned known threats to property as well as lives and therefore the process of leaving a specific structure. The BeSeCu-S might therefore be more appropriate for smaller-scale evacuations (e.g. of a single building or a few properties located close together) rather than larger-scale evacuations (e.g. of whole communities due to nuclear accidents or hurricanes).

Another issue is that the length of the $\mathrm{BeSeCu}-\mathrm{S}$ is considerable, since completing it can take up to 40 minutes. Nevertheless, feedback regarding design and the subdivision of the $\mathrm{BeSeCu}-\mathrm{S}$ into different stages was very positive and people were glad to express some specifics not just once in an overall matter but for specified periods of time (i.e. stages) during the incident. However, it is unclear if this is also applicable for vulnerable groups such as older adults, children or people with cognitive impairments. It must be noted that a representative sample from all countries and regarding all incidents could not be obtained due to the fact that not all incidents occurred in each respective country. A potential self-selection bias needs to be taken into account as well. It is possible that survivors who participated in the study may represent a sub-group of individuals and therefore generalisability of findings 
is unknown. Survivors with severe traumatic experiences might have refused to participate to avoid reminders. Furthermore it needs to be noted that non-response rates are not available for these data.

\section{Conclusion and perspectives}

$\mathrm{BeSeCu}-\mathrm{S}$ is a questionnaire for threatening events and enables the comparison of survivor responses (emotions, cognitions, activities) across different event types, stages of an event and cultures. How stressful is a fire in a public building compared to a domestic fire? Answering questions like this is very difficult since different methodological approaches have been used over the past years. The BeSeCu-S can help advance understanding for researchers and practitioners alike. 


\section{References}

American Psychiatric Association. (2000). Diagnostic and statistical manual of mental disorders (4th ed.). Washington, DC.

Bauer, P. J., Burch, M. M., Scholin, S. E., \& Güler, O. E. (2007). Using cue words to investigate the distribution of autobiographical memories in childhood. Psychological Science, 18(10), 910-6. doi:10.1111/j.1467-9280.2007.01999.x

Bergh Johannesson, K., Stefanini, S., Lundin, T., \& Anchisi, R. (2006). Impact of bereavement among relatives in Italy and Sweden after the Linate airplane disaster. International Journal of Disaster Medicine, 4(3), 110-117. doi:10.1080/15031430600969034

Bornstein, B. H., Liebel, L. M., \& Scarberry, N. C. (1998). Repeated Testing in Eyewitness Memory: A Means to Improve Recall of a Negative Emotional Event. Applied Cognitive Psychology, 12(2), 119-131. doi:10.1002/(SICI)1099-0720(199804)12:2<119::AIDACP500>3.0.CO;2-4

Briere, J., \& Elliott, D. (2000). Prevalence, characteristics, and long-term sequelae of natural disaster exposure in the general population. Journal of Traumatic Stress, 13(4), 661679. doi:10.1023/A:1007814301369

Brown, J. M. (2003). Eyewitness Memory for Arousing Events: Putting Things into Context. Applied Cognitive Psychology, 17(1), 93-106. doi:10.1002/Acp.848

Brunet, A., Weiss, D. S., Metzler, T. J., Best, S. R., Neylan, T. C., Rogers, C., Fagan, J., et al. (2001). The peritraumatic distress inventory: A proposed measure of PTSD criterion A2. The American journal of psychiatry, 158(9), 1480-5. 
Christianson, S.-A. (1992). Emotional stress and eyewitness memory: A critical review. Psychological Bulletin, 112(2), 284-309. doi:10.1037//0033-2909.112.2.284

Christianson, S.-A., \& Loftus, E. F. (1991). Remembering Emotional Events: The Fate of Detailed Information. Cognition \& Emotion, 5(2), 81-108. doi:10.1080/02699939108411027

Collins, D. (2003). Pretesting survey instruments: An overview of cognitive methods. Quality of Life Research, 12(3), 229-38. doi:10.1023/A:1023254226592

Corapcioglu, A., Yargıç, İ., Geyran, P., \& Kocabaşoğlu, N. (2006). Validity and Reliability of Turkish Version of "Impact of Event Scale-Revised” (IES-R). Yeni Symposium, 44(1), 14-22. Retrieved from http://www.yenisymposium.net/fulltext/2006(1)/ys2006_44_1_2.pdf

Cordón, I. M., Pipe, M.-E., Sayfan, L., Melinder, A., \& Goodman, G. S. (2004). Memory for traumatic experiences in early childhood. Developmental Review, 24(1), 101-132. doi:10.1016/j.dr.2003.09.003

Eremenco, S. L., Cella, D., \& Arnold, B. J. (2005). A comprehensive method for the translation and cross-cultural validation of health status questionnaires. Evaluation the health professions, 28(2), 212-232. doi:10.1177/0163278705275342

Field, A. P. (2009). Discovering statistics using SPSS: (and sex and drugs and rock " $n$ " roll) (Third.). London: SAGE.

Fischer, H. W. (2008). Response to disaster. Fact versus fiction and its perpetuation. The sociology of disaster. (Vol. 3). Lanham, Maryland: University Press of America. 
Fothergill, A., Maestas, E. G., \& Darlington, J. D. (1999). Race, Ethnicity and Disasters in the United States: A Review of the Literature. Disasters, 23(2), 156-73. doi:10.1111/1467-7717.00111

Freitag, S., Grimm, A., \& Schmidt, S. (2011). Talking about traumatic events: A crosscultural investigation. Europe's Journal of Psychology, 7(1), 40-61. doi:10.5964/ejop.v7i1.104

Gargurevich, R., Luyten, P., Fils, J.-F., \& Corveleyn, J. (2009). Factor structure of the impact of event scale-revised in two different Peruvian samples. Depression and Anxiety, 26(8), E91-E98. doi:10.1002/da.20430

Glenshaw, M. T., Vernick, J. S., Frattaroli, S., Brown, S., \& Mallonee, S. (2008). Injury Perceptions of Bombing Survivors - Interviews from the Oklahoma City Bombing. Prehospital and Disaster Medicine, 23(6), 500-506.

Gliem, J., \& Gliem, R. (2003). Calculating, Interpreting, and Reporting Cronbach's Alpha Reliability Coefficient for Likert-Type Scales. Midwest Research-to-Practice Conference in Adult, Continuing, and Community Education (pp. 82-88). Columbus, Ohio OR - The Ohio State University.

Grimm, A., Hulse, L., Preiss, M., \& Schmidt, S. (n.d.). Behavioural , emotional and cognitive responses in European disasters: Results of survivor interviews. Disasters.

Grimm, A., Hulse, L., \& Schmidt, S. (2012). Human responses to disasters: A pilot study on peritraumatic emotional and cognitive processing. Europe's Journal of Psychology, 8(1), 112-138. doi:10.5964/ejop.v8i1.303 
Guha-sapir, D. (2010). Statistical Review 2010 Annual Disaster Statistical Review 2010 The numbers and trends. Review - Literature And Arts Of The Americas, 1-42.

Hilton, A., \& Skrutkowski, M. (2002). Translating instruments into other languages: development and testing processes. Cancer Nursing, 25(1), 1-7. doi:10.1097/00002820200202000-00001

Horowitz, M., Wilner, N., \& Alvarez, W. (1979). Impact of Event Scale - A Measure of Subjective Stress. Psychosomatic Medicine, 41(3), 209-218. Retrieved from $<$ Go to ISI>://A1979HB61200004

Hu, L., \& Bentler, P. M. (1999). Cutoff Criteria for Fit Indexes in Covariance Structure Analysis: Conventional Criteria Versus New Alternatives. Structural Equation Modeling, 6(1), 1-55. doi:10.1080/10705519909540118

Hulse, L., Allan, K., Memon, A., \& Read, J. D. (2007). Emotional arousal and memory: a test of the poststimulus processing hypothesis. The American Journal of Psychology, 120(1), 73-90.

Juczyński, Z., \& Ogińska-Bulik, N. (2009). Measurement of post-traumatic stress disorder Polish version of impact of event scale-revised. Psychiatria, 6(1), 15-25.

Kasapoglu, A., \& Mehmet, E. (2004). Comparative Behavioral Response to Future Earthquakes: The Cases of Turkey and USA. Social Behavior and Personality, 32(4), 373-382. doi:10.2224/sbp.2004.32.4.373

Kaysen, D., Morris, M. K., Rizvi, S. L., \& Resick, P. A. (2005). Peritraumatic Responses and their Relationship to Perceptions of Threat in Female Crime Victims. Violence Against Women, 11(12), 1515-1535. doi:10.1177/1077801205280931 
Knuth, D., Kehl, D., \& Schmidt, S. (in press). Development of field study instruments BeSeCu-S and BeSeCu-FR. In S. Schmidt \& E. Galea (Eds.), Behaviour-SecurityCulture. Human behaviour in emergencies and disasters: A cross-cultural investigation. Lengerich: Papst Science Publishers.

Knuth, D., Kehl, D., Stegemann, K., \& Schmidt, S. (in press). Recruitment strategies across all centers. In S. Schmidt \& E. Galea (Eds.), Behaviour-Security-Culture. Human behaviour in emergencies and disasters: A cross-cultural investigation. Lengerich: Papst Science Publishers.

Kobes, M., \& Groenewegen, K. (2009). Consumer fire safety $\square$ : European statistics and potential fire safety measures (Vol. 31).

Kobes, M., Helsloot, I., Vries, B. de, \& Post, J. G. (2010). Building safety and human behaviour in fire: A literature review. Fire Safety Journal, 45(1), 1-11. doi:10.1016/j.firesaf.2009.08.005

Lahad, M., \& Crimando, S. (2010). Preparing for the Next Generation of Disasters. Journal of Jewish Communal Service, 85(2/3), 320-330. Retrieved from http://www.xbrm.com/wpcontent/uploads/2010/11/JJCS_Preparing...Dusasters_SummerFall-2010.pdf

Lindell, M. K., \& Perry, R. W. (2011). The Protective Action Decision Model: Theoretical Modifications and Additional Evidence. Risk Analysis. doi:10.1111/j.15396924.2011.01647.x

Maercker, A., \& Schützwohl, M. (1998). Erfassung von psychischen Belastungssfolgen: Die IES-R -revidierte Version. Diagnostica, 44, 130-141. 
Marmar, C. R., Weiss, D. S., \& Metzler, T. J. (1996). The peritraumatic dissociative experiences questionnaire. In J. P. Wilson \& T. M. Keane (Eds.), Assessing psychological trauma and PTSD (pp. 144-167). New York: The Guilford Press.

McConnell, N. C., Boyce, K. E., \& Shields, T. J. (2009). An Analysis of the Recognition and Response Behaviours of Evacuees of WTC 1 on 9/11. Proceedings 4th International Human Behaviour in Fire Symposium (pp. 659-670). London: interscience communications.

McConnell, N. C., Boyce, K. E., Shields, T. J., Galea, E. R., Day, R. C., \& Hulse, L. (2010). The UK 9/11 evacuation study: Analysis of survivors' recognition and response phase in WTC1. Fire Safety Journal, 45(1), 21-34. doi:10.1016/j.firesaf.2009.09.001

Meyers, L. S., Gamst, G. C., \& Guarino, A. J. (2005). Applied Multivariate Research: Design and Interpretation. Journal of the Operational Research Society (Vol. 45, p. 760). Sage Publications. doi:10.1057/jors.1994.29

Norris, F. H., \& Kaniasty, K. (1992). Reliability of delayed self-reports in disaster research. Journal of Traumatic Stress, 5(4), 575-588. doi:10.1002/jts.2490050407

Ozer, E. J., Best, S. R., Lipsey, T. L., \& Weiss, D. S. (2003). Predictors of posttraumatic stress disorder and symptoms in adults: A meta-analysis. Psychological Bulletin, 129(1), 52-73. doi:10.1037/0033-2909.129.1.52

Petersen, C., Schmidt, S., Power, M., \& Bullinger, M. (2005). Development and pilot-testing of a health-related quality of life chronic generic module for children and adolescents with chronic health conditions: A European perspective. Quality of Life Research, 14(4), 1065-1077. doi:10.1007/s11136-004-2575-z 
Pielmaier, L., \& Maercker, A. (2011). Psychological adaptation to lifethreatening injury in dyads: The role of dysfunctional disclosure of trauma. Psychotraumatology, 2, 8749-61. doi:10.3402/ejpt.v2i0.8749

Pillemer, D. B. (1998). What is remembered about early childhood events? Clinical Psychology Review, 18(8), 895-913. doi:http://dx.doi.org/10.1016/S0272$7358(98) 00042-7$

Preiss, M., Mohr, P., Kopeček, M., Melanová, V., Janečka, V., Rodriguez, M., \& Hájek, T. (2004). Trauma a stress osm měsíců po povodních v roce 2002. Psychiatrie, 8(3), 180186. doi:RIV/00023752: /04:00000426

Proulx, G., \& Reid, I. M. A. (2006). Occupant behavior and evacuation during the Chicago cook county administration building fire. Journal of Fire Protection Engineering, 16(4), 283-309. doi:10.1177/1042391506065951

Rodríguez, H., Quarantelli, E. L., \& Dynes, R. (2006). Handbook of Disaster Research. New York: Springer.

Rubin, D. C. (2000). The distribution of early childhood memories. Memory, 8(4), 265-269. doi:10.1080/096582100406810

Samochine, D. A., Boyce, K. E., \& Shields, T. J. (2005). An Investigation into Staff Behaviour in Unannounced Evacuations of Retail Stores - Implications for Training and Fire Safety Engineering. In I. A. for F. S. Science (Ed.), Fire Safety Science (Vol. 8, pp. 519-530). London: IAFSS Symposium 8. doi:10.3801/IAFSS.FSS.8-519

Schenk, L., Bau, A.-M., Borde, T., Butler, J., Lampert, T., Neuhauser, H., Razum, O., et al. (2006). Mindestindikatorensatz zur Erfassung des Migrationsstatus'. 
1

2

3

4

5

6

7

8

9

10

11

12

13

14

15

16

17

18

19

20

21

22

23

24

25

26

27

28

29

30

31

32

33

34

35

36

37

38

39

40

41

42

43

44

45

46

47

48

49

50

51

52

53

54

55

56

57

58

59

60

Bundesgesundheitsblatt, Gesundheitsforschung, Gesundheitsschutz, 49(9), 853-60. doi:10.1007/s00103-006-0018-4

Stallings, R. A. (2007). Methodological Issues. In H. Rodríguez, E. L. Quarantelli, \& R. Dynes (Eds.), Handbook of Disaster Research (pp. 56-82). New York: Springer Science+Business Media.

Weiss, D. S., \& Marmar, C. R. (1997). The impact of event scale - revised. In J. P. Wilson \& T. M. Keane (Eds.), Assessing psychological trauma and PTSD (pp. 399-411). New York Guilford.

Zhao, C. M., Lo, S. M., Zhang, S. P., \& Liu, M. (2009). A post-fire survey on the preevacuation human behavior. Fire Technology, 45(1), 71-95. doi:10.1007/s10694-007$0040-6$ 


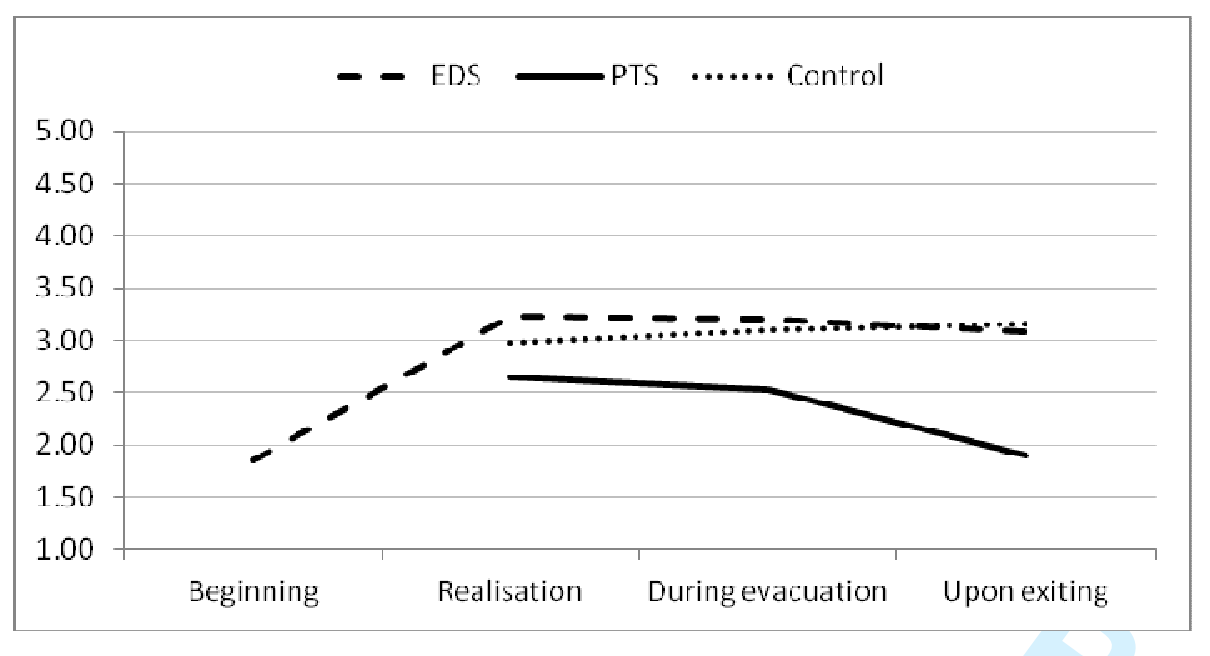

Figure 1. Stage variation of Emotional distress (EDS), Perceived threat (PTS) and perceived control 
Table 1

Sample characteristics $(n=1112)$ with respect to participants' nationality.

\begin{tabular}{|c|c|c|c|c|c|c|c|c|c|c|c|c|c|c|c|c|c|c|}
\hline & \multicolumn{2}{|c|}{ German } & \multicolumn{2}{|c|}{ U.K. } & \multicolumn{2}{|c|}{ Czech } & \multicolumn{2}{|c|}{ Polish } & \multicolumn{2}{|c|}{ Spanish } & \multicolumn{2}{|c|}{ Swedish } & \multicolumn{2}{|c|}{ Turkish } & \multicolumn{2}{|c|}{ Italian } & \multicolumn{2}{|c|}{ Other } \\
\hline & $N$ & $\%$ & $N$ & $\%$ & $N$ & $\%$ & $N$ & $\%$ & $N$ & $\%$ & $N$ & $\%$ & $N$ & $\%$ & $N$ & $\%$ & $N$ & $\%$ \\
\hline Incident & 209 & 100 & 42 & 100 & 166 & 100 & 174 & 100 & 106 & 100 & 79 & 100 & 146 & 100 & 170 & 100 & 15 & 100 \\
\hline Fire in a home & 134 & 64.1 & 17 & 40.5 & 88 & 53.0 & 109 & 62.6 & 40 & 37.7 & 72 & 91.1 & 74 & 50.7 & 43 & 25.3 & 8 & 53.3 \\
\hline Terrorist attack & 0 & 0.0 & 12 & 28.6 & 0 & 0 & 0 & 0 & 29 & 27.4 & 0 & 0 & 5 & 3.4 & 1 & 0.6 & 3 & 20 \\
\hline Flood & 43 & 20.6 & 3 & 7.1 & 63 & 38.0 & 45 & 25.9 & 1 & 0.9 & 0 & 0 & 0 & 0 & 17 & 10 & 0 & 0 \\
\hline Public building fire & 31 & 14.8 & 10 & 23.8 & 15 & 9 & 20 & 11.5 & 36 & 34 & 7 & 8.9 & 30 & 20.5 & 21 & 12.4 & 4 & 26.7 \\
\hline Earthquake & 1 & 0.5 & 0 & 0 & 0 & 0 & 0 & 0 & 0 & 0 & 0 & 0 & 37 & 25.3 & 88 & 51.8 & 0 & 0 \\
\hline Female gender & 116 & 55.5 & 19 & 45.2 & 116 & 69.9 & 93 & 53.4 & 54 & 50.9 & 47 & 59.5 & 50 & 35.5 & 97 & 57.4 & 7 & 46.7 \\
\hline Migrant background & 25 & 12.0 & 10 & 24.4 & 21 & 12.7 & 18 & 10.3 & 15 & 14.4 & 17 & 22.4 & 3 & 2.1 & 11 & 6.5 & 15 & 100 \\
\hline \multicolumn{19}{|l|}{ Relationship status } \\
\hline Single & 42 & 20.2 & 9 & 21.4 & 30 & 18.1 & 39 & 22.5 & 19 & 17.9 & 26 & 33.3 & 34 & 23.8 & 48 & 28.4 & 4 & 26.7 \\
\hline Married & 81 & 38.9 & 23 & 54.8 & 84 & 50.6 & 112 & 64.7 & 52 & 49.1 & 21 & 26.9 & 99 & 69.2 & 42 & 24.9 & 5 & 33.3 \\
\hline Relationship & 70 & 33.7 & 7 & 16.7 & 19 & 11.4 & 10 & 5.8 & 24 & 22.6 & 22 & 28.2 & 0 & 0 & 75 & 44.4 & 1 & 6.7 \\
\hline
\end{tabular}


Table 1 continued

Sample characteristics $(n=1112)$ with respect to participants' nationality.

\begin{tabular}{|c|c|c|c|c|c|c|c|c|c|c|c|c|c|c|c|c|c|c|}
\hline & \multicolumn{2}{|c|}{ German } & \multicolumn{2}{|c|}{ U.K. } & \multicolumn{2}{|c|}{ Czech } & \multicolumn{2}{|c|}{ Polish } & \multicolumn{2}{|c|}{ Spanish } & \multicolumn{2}{|c|}{ Swedish } & \multicolumn{2}{|c|}{ Turkish } & \multicolumn{2}{|c|}{ Italian } & \multicolumn{2}{|c|}{ Other } \\
\hline & $N$ & $\%$ & $N$ & $\%$ & $N$ & $\%$ & $N$ & $\%$ & $N$ & $\%$ & $N$ & $\%$ & $N$ & $\%$ & $N$ & $\%$ & $N$ & $\%$ \\
\hline Divorced & 10 & 4.8 & 3 & 7.1 & 15 & 9 & 4 & 2.3 & 8 & 7.5 & 6 & 7.7 & 6 & 4.2 & 3 & 1.8 & 3 & 20 \\
\hline Widowed & 5 & 2.4 & 0 & 0 & 18 & 10.8 & 8 & 4.6 & 3 & 2.8 & 3 & 3.8 & 4 & 2.8 & 1 & 0.6 & 2 & 13.3 \\
\hline \multicolumn{19}{|l|}{ Qualification } \\
\hline No or lowest & 20 & 9.7 & 2 & 4.8 & 24 & 14.5 & 9 & 5.2 & 20 & 18.9 & 15 & 19.2 & 18 & 12.4 & 4 & 2.4 & 1 & 6.7 \\
\hline Intermediary & 59 & 28.5 & 5 & 11.9 & 34 & 20.5 & 2 & 1.2 & 13 & 12.3 & 4 & 5.1 & 15 & 10.3 & 13 & 7.7 & 1 & 6.7 \\
\hline Higher secondary & 64 & 30.9 & 10 & 23.8 & 68 & 41 & 99 & 57.2 & 25 & 23.6 & 31 & 39.7 & 55 & 37.9 & 68 & 40.5 & 6 & 40 \\
\hline University degree & 64 & 30.9 & 25 & 59.5 & 40 & 24.1 & 63 & 36.4 & 48 & 45.3 & 28 & 35.9 & 57 & 39.3 & 83 & 49.4 & 7 & 46.7 \\
\hline Income $<70 \%$ & 68 & 33.8 & 5 & 11.9 & 78 & 47.3 & 54 & 32.7 & 14 & 14.3 & 17 & 21.8 & 45 & 32.8 & 97 & 62.2 & 3 & 20 \\
\hline $70 \% \geq x \leq 150 \%$ & 91 & 45.3 & 11 & 26.2 & 63 & 38.2 & 93 & 56.4 & 46 & 46.9 & 34 & 43.6 & 48 & 35 & 52 & 33.3 & 12 & 80 \\
\hline Income $>150 \%$ & 42 & 20.9 & 26 & 61.9 & 24 & 14.5 & 18 & 10.9 & 38 & 38.8 & 27 & 34.6 & 44 & 32.1 & 7 & 4.5 & 0 & 0 \\
\hline Age $M(S D)$ & 40.8 & $(15.57)$ & 42.1 & $(13.89)$ & 4.28 & 17.15) & 39.8 & 12.99 & $45.0^{\circ}$ & 12.68 & 47.6 & (20.07) & 37.6 & (11.49) & 32.53 & 13.55 & $5.6^{\prime}$ & (9.53) \\
\hline
\end{tabular}


Table 2

Descriptive statistics and reliabilities for the three scales depending on their stage

\begin{tabular}{lcccccc}
\hline & $\alpha$ & $M$ & $S D$ & Items & a in national & $\alpha$ in incident \\
& & & & & subsamples & subsamples \\
\hline EKS - Beginning & .81 & 15.47 & 6.09 & 7 & $.66-.92$ & $.76-.82$ \\
EDS - Beginning & .90 & 9.26 & 5.17 & 5 & $.70-.96$ & $.75-.93$ \\
EDS - Realisation & .86 & 16.04 & 5.66 & 5 & $.78-.90$ & $.81-.89$ \\
PTS - Realisation & .76 & 5.30 & 2.56 & 2 & $.33, .43, .69-.84$ & $.54, .59, .72-.83$ \\
EDS - During evac. & .89 & 15.86 & 5.89 & 5 & $.82-.91$ & $.79-.92$ \\
PTS - During evac. & .81 & 5.07 & 2.59 & 2 & $.51, .61, .78-.84$ & $.57, .66-.86$ \\
EDS - Upon exiting & .88 & 15.30 & 5.75 & 5 & $.83-.92$ & $.83-.90$ \\
PTS - Upon exiting & .78 & 3.80 & 2.21 & 2 & $.28, .59, .70-.91$ & $.44, .64, .78-.80$ \\
\hline Note. EKS= Emergency knowledge scale; EDS= Emotional distress scale; PTS= Perceived
\end{tabular}


Table 3

Intercorrelations (Spearman-Rho) between pre-, peri- and post-event variables with respect to event stages

\begin{tabular}{|c|c|c|c|c|c|c|c|c|c|c|c|c|c|c|c|}
\hline $1 \mathrm{EKS}^{\mathrm{a}}$ & 1 & 2 & 3 & 4 & 5 & 6 & 7 & 8 & 9 & 10 & 11 & 12 & 13 & 14 & 15 \\
\hline $2 \mathrm{EDS}^{\mathrm{a}}$ & .02 & & & & & & & & & & & & & & \\
\hline $3 \mathrm{PTS}^{\mathrm{b}}$ & $-.07 *$ & $.12 * *$ & & & & & & & & & & & & & \\
\hline $4 \mathrm{EDS}^{\mathrm{b}}$ & $-.23 * *$ & $.18^{* *}$ & $.51^{* *}$ & & & & & & & & & & & & \\
\hline 5 Panic $^{\mathrm{b}}$ & $-.12 * *$ & $.16^{* *}$ & $.41 * *$ & $.60 * *$ & & & & & & & & & & & \\
\hline 6 Control $^{\mathrm{b}}$ & $.31 * *$ & $-.12 * *$ & $-.29 * *$ & $-.45 * *$ & $-.34 * *$ & & & & & & & & & & \\
\hline 7 Evac starting ${ }^{\mathrm{c}}$ & $-.07 *$ & $.13 * *$ & $-.10 * *$ & -.03 & -.01 & -.06 & & & & & & & & & \\
\hline $8 \mathrm{PTS}^{\mathrm{c}}$ & -.06 & $.11 * *$ & $.82 * *$ & $.55^{* *}$ & $.44 * *$ & $-.28 * *$ & $-.13 * *$ & & & & & & & & \\
\hline $9 \mathrm{EDS}^{\mathrm{c}}$ & $-.24 * *$ & $.19 * *$ & $.57 * *$ & $.88 * *$ & $.59 * *$ & $-.46 * *$ & -.04 & $.61 * *$ & & & & & & & \\
\hline $10 \mathrm{Control}^{\mathrm{c}}$ & $.27 * *$ & $-.13 * *$ & $-.32 * *$ & $-.45 * *$ & $-.37 * *$ & $.72 * *$ & $-.07 *$ & $-.35 * *$ & $-.49 * *$ & & & & & & \\
\hline $11 \mathrm{PTS}^{\mathrm{d}}$ & -.07 & $.20 * *$ & $.56^{* *}$ & $.36^{* *}$ & $.37 * *$ & $-.26 * *$ & $-.10 * *$ & $.62 * *$ & $.41 * *$ & $-.28 * *$ & & & & & \\
\hline $12 \mathrm{EDS}^{\mathrm{d}}$ & $-.24 * *$ & $.18^{* *}$ & $.52 * *$ & $.80 * *$ & $.60 * *$ & $-.43 * *$ & $-.08 *$ & $.55^{* *}$ & $.86 * *$ & $-.47 * *$ & $.46 * *$ & & & & \\
\hline 13 Control $^{\mathrm{d}}$ & $.21 * *$ & $-.16 * *$ & $-.29 * *$ & $-.38 * *$ & $-.31 * *$ & $.62 * *$ & -.02 & $-.29 * *$ & $-.41 * *$ & $.72 * *$ & $-.32 * *$ & $-.48 * *$ & & & \\
\hline 14 Weeks since event & $-.13 * *$ & .02 & $.10 * *$ & .00 & .00 & $-.11 * *$ & $.12 * *$ & .03 & .03 & $-.09 *$ & $.11 * *$ & .03 & -.07 & & \\
\hline
\end{tabular}


Table 3 continued

Intercorrelations (Spearman-Rho) between pre-, peri- and post-event variables with respect to event stages

\begin{tabular}{lcccccccccccccccc}
\hline & 1 & 2 & 3 & 4 & 5 & 6 & 7 & 8 & 9 & 10 & 11 & 12 & 13 & 14 & 15 \\
\hline 15 Health & $-.14 * *$ & $.12 * *$ & .05 & $.09 * *$ & $.11^{* *}$ & $-.10^{* *}$ & .00 & .04 & $.08^{*}$ & $-.09^{*}$ & $.08^{*}$ & $.10^{* *}$ & $-.17^{* *}$ & -.03 & \\
16 IES-R & $-.10^{* *}$ & $.22 * *$ & $.47 * *$ & $.57 * *$ & $.49 * *$ & $-.35^{* *}$ & .03 & $.52^{* *}$ & $.64 * *$ & $-.39 * *$ & $.43^{* *}$ & $.62^{* *}$ & $-.40^{* *}$ & -.03 & $.18^{* *}$
\end{tabular}

Note. $* p<.05 * * p<.01 . ;{ }^{\mathrm{a}}=$ Beginning $;{ }^{\mathrm{b}}=$ Realisation; ${ }^{\mathrm{c}}=$ During Evacuation; ${ }^{\mathrm{d}}=$ Upon Exiting; Health $=$ current health status from 1

(very good) - 5 (very bad); Evac starting: 1=within $30 \mathrm{sec}-6=$ more than $30 \mathrm{~min}$ 
Table 4

Initial activities with respect to the time taken to begin evacuating and injuries incurred

\begin{tabular}{|c|c|c|c|c|c|c|c|c|}
\hline \multirow[b]{3}{*}{ Initial activities } & \multicolumn{6}{|c|}{ Evacuation starting time } & \multicolumn{2}{|c|}{ Injuries } \\
\hline & $\leq 30 \mathrm{sec}$ & $\leq 2 \min$ & $\leq 5 \min$ & $\leq 10 \mathrm{~min}$ & $\leq 30 \mathrm{~min}$ & $>30 \mathrm{~min}$ & Yes & No \\
\hline & $N(\%)$ & $N(\%)$ & $N(\%)$ & $N(\%)$ & $N(\%)$ & $N(\%)$ & $N(\%)$ & $N(\%)$ \\
\hline Nothing for a while & $8(15.4)$ & $25(16.9)$ & $20(12.9)$ & $23(17.3)$ & $16(17.8)$ & $16(12.6)$ & $13(14.8)$ & $143(15.7)$ \\
\hline Alert/save others & $15(28.8)$ & $44(29.7)$ & $42(27.1)$ & $36(27.1)$ & $23(25.6)$ & $27(21.3)$ & $25(28.4)$ & $216(23.7)$ \\
\hline Inform others & $2(3.8)$ & $5(3.4)$ & $5(3.2)$ & $5(3.8)$ & $3(3.3)$ & $6(4.7)$ & $4(4.5)$ & $41(4.5)$ \\
\hline Sought help from emergency services & $3(5.8)$ & $15(10.1)$ & $17(11.0)$ & $19(14.3)$ & $15(16.7)$ & $5(3.9)$ & $7(8.0)$ & $103(11.3)$ \\
\hline Sought shelter inside & $0(0.0)$ & $6(4.1)$ & $6(3.9)$ & $5(3.8)$ & $4(4.4)$ & $2(1.6)$ & $6(6.8)$ & $21(2.3)$ \\
\hline Protect my property & $1(1.9)$ & $4(2.7)$ & $7(4.5)$ & $6(4.5)$ & $4(4.4)$ & $31(24.4)$ & $3(3.4)$ & $75(8.2)$ \\
\hline Gave up & $0(0.0)$ & $2(1.4)$ & $1(0.6)$ & $6(4.5)$ & $3(3.3)$ & $2(1.6)$ & $0(0.0)$ & 17(1.9) \\
\hline Gathered items & $4(7.7)$ & $17(11.5)$ & $19(12.3)$ & $13(9.8)$ & $7(7.8)$ & $21(16.5)$ & $6(6.8)$ & $85(9.3)$ \\
\hline Sought information & $6(11.5)$ & $13(8.8)$ & $16(10.3)$ & $8(6.0)$ & $5(5.6)$ & $7(5.5)$ & $0(0.0)$ & $106(11.6)$ \\
\hline Other & $13(25.0)$ & $17(11.5)$ & $22(14.2)$ & $12(9.0)$ & $10(11.1)$ & 10(7.9) & $24(27.3)$ & $104(11.4)$ \\
\hline
\end{tabular}


Table 5

Initial activities with respect to pre-event and other peri-event variables

\begin{tabular}{|c|c|c|c|c|c|c|c|c|c|c|c|c|c|c|}
\hline \multirow[b]{2}{*}{ Initial activities } & \multicolumn{2}{|c|}{ EKS } & \multicolumn{2}{|c|}{ EDS } & \multicolumn{2}{|c|}{ PTS } & \multicolumn{2}{|c|}{ Panic } & \multicolumn{2}{|c|}{ Control } & \multicolumn{2}{|c|}{ Time pressure } & \multicolumn{2}{|c|}{ Reaction } \\
\hline & $M$ & $S D$ & $M$ & $S D$ & $M$ & $S D$ & $M$ & $S D$ & $M$ & $S D$ & $\begin{array}{c}Y e s \\
N(\%)\end{array}$ & $\begin{array}{c}N o \\
N(\%)\end{array}$ & $\begin{array}{c}\text { Automatic } \\
N(\%)\end{array}$ & $\begin{array}{c}\text { Conscious } \\
N(\%)\end{array}$ \\
\hline Nothing for a while & 1.93 & 0.71 & 3.52 & 1.12 & 3.00 & 1.36 & 2.33 & 2.14 & 2.47 & 1.20 & $104(13.4)$ & $53(22.3)$ & $92(20.0)$ & 63(11.6) \\
\hline Alert/save others & 2.40 & 0.89 & 3.14 & 1.04 & 2.77 & 1.22 & 1.62 & 1.71 & 3.23 & 1.13 & $210(27.1)$ & $35(14.7)$ & $116(25.2)$ & $129(23.7)$ \\
\hline Inform others & 2.06 & 0.83 & 2.96 & 1.14 & 1.91 & 1.16 & 0.98 & 1.34 & 3.25 & 1.04 & $27(3.5)$ & $18(7.6)$ & $13(2.8)$ & $33(6.1)$ \\
\hline Sought help from emergency services & 2.12 & 0.84 & 3.54 & 1.09 & 2.50 & 1.21 & 2.29 & 2.56 & 2.55 & 1.17 & $105(13.6)$ & $9(3.8)$ & $58(12.6)$ & $54(9.9)$ \\
\hline Sought shelter inside & 2.38 & 1.03 & 3.75 & 1.13 & 3.46 & 1.40 & 2.85 & 2.51 & 3.11 & 1.05 & $22(2.8)$ & $5(2.1)$ & $13(2.8)$ & $14(2.6)$ \\
\hline Protect my property & 2.14 & 0.78 & 3.10 & 1.15 & 2.18 & 1.15 & 1.58 & 1.92 & 3.03 & 1.09 & $66(8.5)$ & $13(5.5)$ & $38(8.2)$ & $41(7.5)$ \\
\hline Gave up & 2.18 & 0.96 & 3.24 & 1.23 & 3.06 & 1.52 & 1.81 & 1.83 & 2.00 & 1.17 & $14(1.8)$ & $3(1.3)$ & $11(2.4)$ & $6(1.1)$ \\
\hline Gathered items & 2.11 & 0.82 & 3.13 & 1.08 & 2.58 & 1.26 & 1.76 & 2.18 & 3.17 & 1.09 & $69(8.9)$ & $23(9.7)$ & $34(7.4)$ & $58(10.7)$ \\
\hline Sought information & 2.50 & 0.92 & 2.84 & 1.12 & 2.19 & 1.11 & 1.14 & 1.82 & 3.38 & 1.13 & $61(7.9)$ & $46(19.3)$ & $28(6.1)$ & $77(14.2)$ \\
\hline Other & 2.22 & 0.93 & 3.09 & 1.17 & 2.63 & 1.38 & 1.83 & 2.21 & 2.97 & 1.24 & $96(12.4)$ & $33(13.9)$ & $58(12.6)$ & $69(12.7)$ \\
\hline
\end{tabular}

